\title{
Effects on Hemodynamic Enhancement and Discomfort of a New Textile Electrode setup during Calf Neuromuscular Electrical Stimulation
}

\section{Sundström}

Karolinska Institutet

R Juthberg

Karolinska Institutet

J Flodin

Karolinska Institutet

L Guo

University of Borås

Paul W. Ackermann ( $\nabla$ paul.ackermann@ki.se )

Karolinska University Hospital

\section{Research Article}

Keywords: Electric Stimulation Therapy, textile electrodes, Motor point, NMES, hemodynamics, pain

Posted Date: January 18th, 2022

DOI: https://doi.org/10.21203/rs.3.rs-1199890/v1

License: (c) (1) This work is licensed under a Creative Commons Attribution 4.0 International License. Read Full License 


\section{Abstract}

Background: Neuromuscular electrical stimulation (NMES) may improve venous hemodynamics to decrease the risk of venous thromboembolism. However, whether calf-NMES using textile electrodes integrated in socks can improve venous hemodynamics without discomfort is unknown.

Aim: The primary aim of this study was to examine whether calf-NMES, using one new textile electrodecompared to an established gel electrode-setup, could induce significant dose-dependent increases in the peak venous velocity (PVV) and non-inferior comfort.

Methods: On ten healthy participants, intensity (mA) of calf-NMES (frequency $36 \mathrm{~Hz}$, phase duration $200 \mu s$ ) was increased until a visible plantar flexion (threshold 1), plus an additional six NMES-levels (threshold II), utilizing two different electrode setups. A new transverse textile electrode (TTE) setup consisted of two textile electrodes $(2 \times 2.5 \mathrm{~cm})$ sewn into the back of a sock placed $2 \mathrm{~cm}$ apart at the largest circumference of the calf. The established gel electrodes $(3 \times 3 \mathrm{~cm})$ were placed on the participants best motor points and designated the motor point electrode (MPE) setup. PVV was measured in v. poplitea and v. femoralis at baseline and at threshold I and I/ using a Doppler ultrasound machine. Comfort was assessed with a numerical rating scale (NRS, 0-10). Values were expressed as medians and significance was set to $p<0.05$.

Results: In v. poplitea both TTE and MPE induced significant dose-dependent increases in PVV from baseline to threshold / with $123 \%$ and $63 \%$ and to threshold I/ with $319 \%$ and $208 \%$, respectively (all p 0.001$)$. TTE, however, resulted in significantly higher increase of PVV in v. poplitea than MPE. In V. femoralis both TTE and MPE induced significant dose-dependent increases in PVV from baseline to threshold / with $47 \%$ and $55 \%$ and to threshold I/ with $102 \%$ and $107 \%$, respectively (all p<0.001). In v. femoralis, however, there were no significant differences in PPV between TTE and MPE $(p=0.700)$. MPE compared to TTE resulted in lower NRS, 1 versus 2, at threshold I $(\mathrm{p}<0.001)$, however at threshold II there was no difference in NRS $(p=0.836)$.

Conclusion: A new reusable TTE produces non-inferior dose-dependent venous hemodynamics compared to a standard MPE. Further development, however, is needed concerning textile electrode discomfort to improve patient compliance.

Trial registration: Retrospectively registered with ISRCTN registry. Trial ID: ISRCTN49260430. Date: $11 / 01 / 2022$.

\section{Introduction}

Venous stasis is one of the major causes of venous thromboembolism (VTE). VTE-prevention targeting venous stasis of the calf include mechanical interventions, such as neuromuscular electrical stimulation (NMES), which today have limited effect due to poor patient compliance (1). Calf-NMES could potentially be integrated into socks resulting in better mobility and thereby improved patient compliance. Although 
textile electrodes have demonstrated good function in electrostimulation $(2,3)$, there is to the best of our knowledge no prior studies comparing the venous hemodynamic effects of reusable textile electrodes to traditional NMES-gel electrodes.

In order to address the above issues, we propose the creation of a NMES sock. However, the textile electrodes in a NMES sock have to produce dose-dependent increases in the peak venous velocity (PVV) of both v. poplitea and v. femoralis, which have been associated with an efficient VTE-preventive profile $(1,4)$. Moreover, the textile electrodes must show non-inferior PVV as compared to ordinary gel electrodes.

A standard motor point electrode (MPE) setup of gel-electrodes has demonstrated reduced discomfort compared to random electrode placement (5-7). Whether textile electrodes in fixed positions according to a motor point map (8), i.e. a transverse textile electrode (TTE) setup, demonstrate non-inferior comfort compared to traditional MPE on the calf has not been investigated.

We hypothesized that TTE would render in non-inferior hemodynamics and comfort compared to MPE. Thus, the primary aim of this study was to examine whether calf-NMES, using one new TTE- and one established MPE-setup, both could induce significant dose-dependent increases in the PVV in v. poplitea and $\mathrm{v}$. femoralis related to the current intensity applied. The secondary aim of this study was to explore whether TTE could produce non-inferior hemodynamics and discomfort compared to the established MPE.

\section{Materials And Methods}

\section{Subjects}

Ten healthy individuals (five men and five women) were included in the study. All subjects completed a questionnaire on subject characteristics before entering the study (Table 1), including measurements to calculate body fat using the US Navy formula (9) and an estimation of their physical activity on a scale of 1-6 using the Grimby/Frändin activity scale (10). Subjects aged between 18-75 years were eligible for inclusion. The exclusion criteria were pregnancy, skin ulcers, previous surgery on blood vessels of the lower limbs, pacemaker, intracardiac defibrillator, advanced heart disease, kidney failure and neuromuscular or metabolic disease.

Table 1. Subject characteristics $(n=10)$. 


\begin{tabular}{ll} 
Variable & Median (Range) \\
\hline Age (years) & $27(24-55)$ \\
\hline Height $(\mathrm{cm})$ & $174(164-187)$ \\
\hline Weight $(\mathrm{kg})$ & $64(56-110)$ \\
\hline BMI $\left(\mathrm{kg} / \mathrm{m}^{2}\right)$ & $22(19-32)$ \\
\hline Body fat $(\%)$ & $20(9-30)$ \\
\hline Waist circumference $(\mathrm{cm})$ & $73(67-114)$ \\
\hline PAS, range 1-6 & $5(3-6)$
\end{tabular}

$\mathrm{M}=$ median, $\mathrm{R}=$ Range. $\mathrm{BMI}=$ body mass index, $\mathrm{PAS}=$ Physical activity scale .

\section{Electrode-Setups}

In this study, two different electrode setups for applying NMES to the calf were tested and compared regarding various outcomes. The electrode setups differed regarding electrode- type, -size and placement.

The first electrode setup, designated as the transverse textile electrode (TTE) setup, consisted of two rectangular textile electrodes $(2 \times 2.5 \mathrm{~cm})$ transversally woven into the back of a sock (Polyamide/Lycra blended yarn). The electrodes were placed approximately at the largest circumference of the calf, and with the electrodes inner edges equally distanced from the midline of the calf approximately $2 \mathrm{~cm}$ apart (Fig. 1). The electrodes were woven into the sock using so called intarsia knitting which allows for seamless integration of patterns of functional components in a single process. The material of the electrodes was silver coated polyamide multifilament yarn, with trade name Shieldex ${ }^{\circledR}$ (produced by Statex Produktions und Vertriebs $\mathrm{GmbH}$ ). On the outside of the sock the electrodes medial and lateral edges were covered with elastic yarn floats (Polyamide/Lycra). The purpose of the floats was to hold a moisture-containing $(2 \mathrm{ml} \mathrm{NaCl} 0.9 \mathrm{mg} / \mathrm{ml})$ melamine sponge $(0.5 \times 2 \times 2.5 \mathrm{~cm})$ in direct contact with each of the underlying electrodes, in order to increase the local pressure and humidity of the electrode/skininterface, and thus uphold an adequate electrical conduction (11).

The second electrode setup utilized commercially available standard adhesive gel electrodes (Compex Snap, Performance, DJO Global, USA, $5 \times 5 \mathrm{~cm}$ ) manually trimmed to squares sized $3 \times 3 \mathrm{~cm}$ to reduce the current intensity needed to induce a muscle response. The standard electrodes were placed on the skin areas of the calf, one on the medial side and one the lateral side, that required the least NMES current intensity to trigger a calf muscle response, i.e. the "best" motor points (MP). This electrode setup was designated the motor point electrode (MPE) setup.

\section{Motor point scan}


The best MPs were found by scanning one half of the calf at a time (medial/lateral), using the NMES device's $3 \mathrm{~Hz}$ sinusoidal wave motor scan program, Chattanooga Physio (DJO Nordic, Malmoe, Sweden). Prior to the MP scan, the side of the calf about to be scanned was covered by a thin layer of conductive gel. A reference electrode (Compex Snap, Performance, DJO Global, USA, $5 \times 5 \mathrm{~cm}$ ) was placed on the contralateral side from the MP scan over the largest circumference of the calf, on a distance from the calf's midline corresponding to $15 \%$ of the calf's largest circumference. The MP scan started at NMESlevel 1 during which time the MP scan pen was used to search through one whole side of the posterior calf in accordance with the NMES-device instruction. If no motor point was found, the current intensity was increased by one NMES-level with a subsequent re-scan. This procedure was then repeated until a visible muscle twitch was detected in the calf, indicating the location of a MP, which was subsequently marked out on the scanned side (medial or lateral).

\section{NMES-Settings}

NMES was applied to the skin via electrodes using a DJOTM Chattanooga Physio NMES device with a biphasic, symmetric square wave. Based on previous studies on NMES discomfort, frequency was set to $36 \mathrm{~Hz}$, phase duration $200 \mu \mathrm{s}$, ramp up time $0.5 \mathrm{~s}$ and ramp down time $0.25 \mathrm{~s}$ (12). The muscle rest between each stimulation (OFF-time) was $8 \mathrm{~s}$. The duration of stimulation between each ramp up and ramp down time, referred to as the plateau time, was varied for different tests between $0.5 \mathrm{~s}, 1.5 \mathrm{~s}$, $3 \mathrm{~s}$ and $5 \mathrm{~s}$. The order in which the plateau times were tested was randomised. The NMES-level (0-999), representing a non-linear relationship to the current intensity ranging from 0-120 milliampere $(\mathrm{mA})$, was gradually increased one NMES-level at a time as described below.

\section{NMES Threshold I \& II}

When testing the two electrode setups, outcomes were registered at two distinct current intensities, designated threshold I and threshold II. For every test performed, the current intensity was slowly increased one NMES-level at a time until a visible plantar flexion was induced. The current intensity needed to induce this plantar flexion was defined as threshold I. Visible plantar flexion was chosen as a point for outcome measure because, 1 ) it can be dichotomised; either you can see a plantar flexion, or you cannot, and 2) it will likely increase PVV (13). The same examiner determined for all participants when a visible plantar flexion was induced by the NMES, thus avoiding any examiner bias. Threshold II was defined as the current intensity corresponding to threshold / plus an additional six NMES-levels increase on the NMES-device. For example, if plantar flexion was induced at NMES-level 20 (threshold I), threshold //would correspond to NMES-level $26(=20+6)$. Note that since different subjects required different current intensities to produce a plantar flexion, threshold I and threshold I/ could represent different current intensities for different subjects.

\section{Hemodynamic measurements}

Using a Philips CX50 (2013) Doppler ultrasound machine (Philips Medical Systems, Andover, MA, USA), the widest accessible part of $v$. poplitea and v. femoralis was located and visualised in a longitudinal 
section plane before beginning the hemodynamic measurements. For the two electrode setups (TTE \& MPE), PVV was measured in v. poplitea and v. femoralis at baseline (i.e. electrodes attached but no NMES applied), at threshold I, and at threshold II. For each subject in the study, all hemodynamic measurements were performed on v. poplitea and v. femoralis respectively, by one and the same ultrasonographist. PVV was registered by the ultrasonographist using the ultrasound machines measuring tool. For each different electrode setup, PVV was always measured during three consecutive stimulations, enabling the collection of an average PVV value for the statistical analysis, thus reducing the effect of potential measuring errors. After analysing the refill time of the veins, an eight second OFF-time was decided to be used between plateau times for the veins to be adequately refilled with blood before the next upcoming muscle contraction and PVV measurement. To quantify the potential benefit of NMES versus the resting state, for each subject and setting, the percentual increase in PVV at threshold I and threshold II, as compared to PVV at baseline, was calculated. The formula used to calculate the percentual increase was $($ Percentual increase $)=(((P V V$ with stimulation $)-($ PVV at baseline $)) /($ PVV at baseline $)) \times 100$.

\section{Discomfort}

For each stepwise increase in NMES-level when testing the two electrode setups, subjects were asked to fill in a form to rate their discomfort on a numerical rating scale (NRS) $0-10$, where 0 was described to the subject as no discomfort and 10 as the worst imaginable discomfort or pain (14).

\section{Statistical analysis}

The sample size was determined prior to the start of the experiment based on a pilot study with the significance level set at $\mathrm{p}<.05$ and power at $80 \%$ regarding the primary outcome, hemodynamics, measured with the PVV in v. poplitea. Based on the calculations, ten subjects were needed to find a significant increase in PVV with an increase of the current intensity from threshold / to II. We set the final sample size to ten subjects.

The data was analyzed using SPSS version 27 (IBM Corp. Released 2016. IBM SPSS Statistics for Windows, Armonk, NY: IBM Corp.) in cooperation with a statistician. The four tested plateau times did not demonstrate any statistically significant difference regarding PVV or NRS, regardless of the electrode setup or threshold. For this reason, the outcome values for PVV and NRS used in the final statistical analysis were based on all values, for all subjects, obtained during the four different plateau times. Based on the relatively small number of subjects and the Shapiro-Wilk indicating non normal distribution, the non-parametric Wilcoxon signed rank test was chosen to determine if there were any statistically significant differences between medians regarding current intensity, PVV or NRS for the two electrode setups. The data included some outliers, which were handled both by using a rank-based statistics test and by adjusting the values of the outliers to the lowest/highest value within 1.5 times the interquartile range from the first respectively third quartile for the inferential statistics (15). Analysis of correlations between subject characteristics and collected data was performed using Spearman's $\rho$. Data is presented with median, min-max range and when comparisons were made, $p$-value. The significance level for all 
analyses was set to $p<0.05$. For the remainder of the text, "statistically significant" will be shortened as "significant".

\section{Results}

\section{Current intensities for calf-NMES}

The current intensity required to reach threshold I, was significantly lower when using MPE, 16mA (7.5$24 \mathrm{~mA})$, as compared to TTE, $26 \mathrm{~mA}(20-44.5 \mathrm{~mA})(\mathrm{p}=0.005)$. To reach threshold II, the MPE setup also required a significantly lower current intensity, $21 \mathrm{~mA}(17-27.5 \mathrm{~mA})$, compared to the TTE setup, $29.5 \mathrm{~mA}$ $(24-46 \mathrm{~mA})(\mathrm{p}=0.005)($ Fig. 2).

\section{Hemodynamics of calf NMES}

\section{Hemodynamics in v. poplitea}

The baseline PVV in v. poplitea was, median (range), $14.3 \mathrm{~cm} / \mathrm{s}(9.8-24.0 \mathrm{~cm} / \mathrm{s})$. There was a significant increase of PVV with 123\% (30 - 500\%) from baseline to threshold l, when using TTE ( $<<0.001)$. MPE also exhibited a significant increase of PVV with $63 \%((-1)-352 \%)$ from baseline $(p<0.001)$. The TTE setup resulted in a significantly higher percentual increase of PVV in v. poplitea than the MPE setup $(p=0.005)$ (Fig. 3).

Increasing the current intensity to threshold I/ resulted in a significant increase of PVV from baseline of $319 \%$ (59 - 766\%), when using TTE ( $<<0.001)$. When increasing the current intensity to threshold I/ using MPE, a significant increase of PVV with $208 \%(12-561 \%)$ from baseline was observed $(p<0.001)$. The increase of PVV from baseline was significantly greater at threshold II as compared to threshold I, for both the TTE and the MPE setups ( $\mathrm{p}<0.001)$ (Fig 1A). At threshold I/ the TTE setup resulted in a significantly higher percentual increase of PVV in v. poplitea, as compared to baseline, than the MPE setup ( $p=0.026)$ (Fig. 3).

\section{Hemodynamics in v. femoralis}

The baseline PVV in v. femoralis was $14.2 \mathrm{~cm} / \mathrm{s}(10.4-21.0 \mathrm{~cm} / \mathrm{s})$. There was a statistically significant increase of PVV with 47\% ((-4) - 447\%) from baseline to threshold / when using TTE ( $<<0.001)$. When using MPE there was also a significant increase of PVV in v. femoralis with 55\% ((-12) - 168\%) from baseline to threshold I $(\mathrm{p}<0.001)$. The percentual increase of PVV in v. femoralis when comparing the TTE and MPE setups did not differ significantly $(p=0.635)$ (Fig 4).

Increasing the current intensity to threshold I/ resulted in a significant increase of PVV with $102 \%((-8)$ $491 \%)$ from baseline, when using TTE $(\mathrm{p}<0.001)$. Also, when using MPE at threshold II a significant increase of PVV with 107\% (0 - $409 \%$ ) from baseline was observed. The increase of PVV from baseline was significantly greater at threshold I/ compared to threshold I, both when using TTE and MPE $(\mathrm{p}<0.001)$ 
(Fig 1B). At threshold I/ there was no significant difference in increase of PVV between TTE and MPE $(p=0.700)$ (Fig. 4).

\section{Discomfort of calf NMES}

Using MPE resulted in a statistically significantly lower NRS, $1(0-3)$, compared to the use of TTE, NRS 2 $(0-7)$, at threshold I $(\mathrm{p}<0.001)$. At threshold II, the two electrode setups did not demonstrate any significant difference in NRS $(p=0.836)$. However, at threshold II, subjects reported significantly higher NRS with both types of electrode setups compared to threshold I $(p<0.001)$ (Fig. 5).

\section{Associations of subject characteristics with outcome}

Female sex was significantly associated with a higher current intensity $(\mathrm{mA})$ required to produce a plantar flexion, both with TTE and MPE $(p=0.005, p=0.001)$. Female gender was also associated with a higher body fat percentage $(p=0.014)$. None of the parameter's age, gender, $B M I$, body fat percentage or waist circumference was significantly correlated with NRS or the percentual increase of PVV in v. poplitea or v. femoralis.

\section{Discussion}

The main finding of this study was that the PVV in both v. poplitea and v. femoralis exhibited a doseresponse relationship with the increased current intensity of calf NMES using either TTE or MPE. The TTE setup demonstrated superior hemodynamics compared to the MPE setup in v. poplitea at the lowest current intensity that could induce a plantar flexion (threshold I). The MPE setup, however, required a lower current intensity and resulted in less discomfort to elicit a plantar flexion of the ankle. Aside from differences in electrode setups, a higher current intensity was needed for female participants to perform a plantar flexion of the ankle.

The finding that the PVV in both v. poplitea and v. femoralis exhibited significant median increases of 50$100 \%$ from baseline to threshold / conform with results from earlier studies on NMES $(4,16)$. However, to the best of our knowledge this is the first study demonstrating improved venous hemodynamic effects of reusable textile electrodes. Moreover, the observation that these venous flows could be enhanced an additional 2-3-times, both with MPE and TTE, by increasing the current intensity of the NMES by a mean of 1.5-6.5 mA, suggests a dose-dependent response between the applied current intensity and the PVV produced.

The finding of a dose-dependent relationship between the current intensity of applied NMES $(36 \mathrm{~Hz})$ and increased venous return is supported by the literature, but novel when it comes to textile electrodes as well as assessments of blood flow in v. poplitea and v. femoralis in the same individual. An earlier study strengthens our finding by demonstrating a relationship between the current intensity applied via NMES (frequencies: 1, 3 and $5 \mathrm{~Hz}$ ) and increased venous velocity in v. femoralis (17). Moreover, another study corroborates our observations in v. poplitea by demonstrating that different subjects, who used NMES 
with various current intensities (frequency: $36 \mathrm{~Hz}$ ) exhibited PVV that on a group level were dosedependent to the electrical current (18).

The presumable explanation to the observed dose-dependence between current intensity and increased PVV lies in the earlier knowledge that there is a linear relationship between the current intensity and muscle torque production (19). Thus, a higher current intensity will cause more muscle fibers to contract (20), which in turn increases the squeezing of the calf veins, filled with approximately 100-150 ml blood (21), subsequently inducing enhanced PVV (17). An additional explanation to the observed increase in PVV may be that higher current intensities induces an activation of the sympathetic nervous system causing a "fight and flight" response with contraction of the blood vessel circumference causing increased blood flow velocity (22).

A dose-dependent increase in venous blood flow velocity using calf-NMES, and especially the new textile electrodes, is important for the prevention of deep vein thrombosis (DVT) and venous thromboembolism by reducing the stasis $(1,4)$. The minimum hemodynamic effect of NMES to prevent development of DVT is unknown. Studies on hemodynamics during voluntary activation of the muscle pump may however provide comparable data, which demonstrate at least a twofold increase in PVV $(13,23,24)$. Thus, our observed 2-3-fold increases in PVV in v. poplitea and v. femoralis may reflect clinically relevant enhancements of venous velocity for preventing DVT.

The second most important finding of this study was the identification that the TTE setup produced a better hemodynamic response in v. poplitea compared to the MPE setup. This observation was astonishing and necessitates replication but may have implications when deciding which electrodes to use and how to place electrodes to produce an optimal venous return in order to prevent DVT. The mechanism behind this observed hemodynamic effect could have several explanations.

The most reasonable explanation to the enhanced hemodynamic response seen with TTE setup is the related finding that the TTE setup required a higher current intensity to produce a plantar flexion of the ankle as compared to the MPE setup. The degree of plantar flexion could, however, be determined to not be different between the two electrode setups since the same examiner examined the plantar flexion with both electrode setups. The higher electrical current with TTE setup may however also induce indirect, local effects on blood vessels via the nervous system (25). Thus, it has been demonstrated that electrical stimulation causes sympathetic nerve fiber activation, which may locally constrict $v$. poplitea with subsequent increase in blood flow velocity (22).

The conception that higher electrical current may induce a local nervous system mediated constriction of v. poplitea is supported by another observation. The finding that TTE setup produced a better hemodynamic response in v. poplitea, but not in v. femoralis, as compared to the MPE setup suggests that the higher electrical current rather induced local changes, such as constriction of v. poplitea. Such a conclusion would also be in line with the idea that higher electrical current at a certain level cannot produce more blood flow via muscle contraction, but rather via local sympathetic activation (22). This hypothesis, however, requires further research. 
Another explanation to the elevated hemodynamic response observed with the TTE setup as compared to the MPE setup may be that the differences in PVV are dependent on the placement of the electrodes on the muscle bulk and subsequent degree of muscle activation. The TTE setup was configured with electrodes transversely, approximately at the thickest portions of both the lateral and medial gastrocnemius muscles, suggesting a good muscle contraction to activate venous return (26). The MPE setup, on the other hand, depended on the subject's best motor points to trigger a plantar flexion of the ankle. Thus, the MPE setup often resulted in a longitudinal placement of electrodes, which may suggest a less beneficial muscle stimulation to compress the veins of the calf. The explanation that the two electrode setups elicit differential muscle-vein pump effects does however not clarify why the PVV with the TTE setup compared with the MPE setup was better only in v. poplitea and not in v. femoralis. Further studies should explore how different electrode placements on the calf may optimize the effect of the muscle-vein pump and whether these effects are related to direct dose-dependent muscular contraction or related to indirect activation of the nervous system.

Another aspect to consider when deciding the optimal placement of electrodes is the discomfort of the patients. The observation that the MPE setup exhibited less discomfort at threshold / as compared to the TTE setup suggests a better compliance with treatment when electrodes are placed on motor points, which is in line with earlier studies (6). The finding of no difference in discomfort between the MPE and TTE setup at threshold II, on the other hand, suggests that discomfort always should be evaluated together with the hemodynamic effect to optimize patient outcome. To find an optimal placement of electrodes and a tolerable, yet hemodynamically effective, level of electrical current is the million-dollar question, which from the results of this study seem to be a very individual matter. The great variations in PVV observed, both at baseline and with calf-NMES, are suggestive of vast individual variations. Variations of PVV are, however, known from earlier studies (27).

This study also investigated the subject characteristics in relation to the outcome variables. The observation that females required higher current to obtain a plantar flexion of the ankle suggests that differences in subcutaneous fat composition may impede the flow of the electrical current, as has been suggested before (28). The differences between genders seen in electrical current required did, however not cause dissimilarities in discomfort or in hemodynamics, suggesting that both sexes may benefit equally from calf-NMES.

Another aspect that might be of importance to the patient is the potential cost-reduction of reusable electrodes could provide. Textile electrodes that are integrated in a sock could make the electrode placement easier, even for new users, and the reusability of the textile will significantly reduce the operational costs (i.e. costs of the electrodes).

A possible limitation of the study is the relatively low number of participants, which hinders conclusions especially on associations between subject characteristics and outcome. This study focused the hemodynamic analyses on PVV, while omitting other hemodynamic variables, the main reason being that PVV has been considered the main factor related to the outcome of DVT (29). The strengths of the study 
include the fact that hemodynamic assessments were performed in both v. poplitea and v. femoralis with two types of electrode setups and investigated the factor of perceived discomfort.

\section{Conclusion}

Neuromuscular electrical stimulation of the calf increases peak venous velocity in a dose-dependent and clinically relevant manner in both $\mathrm{v}$. poplitea and $\mathrm{v}$. femoralis using both a transverse-textile- and a motor point electrode setup. Motor point electrode setup decreases the need for electrical current and reduces discomfort. Transverse-textile electrode setup, however, seems to enhance peak venous velocity in v. poplitea to a higher extent, and with yet not fully explored mechanisms, as compared to motor point electrode setup. A new reusable transverse-textile electrode is non-inferior regarding venous hemodynamics compared to standard single-use adhesive electrodes. There is, however, a need of further development in reducing the discomfort of textile electrodes in order to improve patient compliance.

\section{Abbreviations}

DVT - Deep Vein Thrombosis

MP - Motor Point

MPE - Motor Point Electrode

NMES - Neuromuscular Electrical Stimulation

NRS - Numerical Rating Scale

PVV - Peak Venous Velocity

TTE - Transverse Textile Electrode

VTE - Venous Thromboembolism

\section{Declarations}

\section{Ethics approval and consent to participate}

Ethical approval was obtained from the Regional Ethical Review Committee in Stockholm (Dnr: 201904020).

All subjects signed an informed consent before being enrolled in the study.

\section{Consent for publication}

There are no photographs of persons in this manuscript that require consent for publication. 
Availability of data and materials

The datasets used and/or analysed during the current study are available from the corresponding author on reasonable request.

\section{Competing interests}

The authors declare that they have no competing interests.

\section{Funding}

This work was supported by the strategic innovation programs Swelife and Medtech4Health, which are jointly arranged and funded by Sweden's Innovation Agency (Vinnova), Formas and Energimyndigheten.

\section{Authors' contributions}

PA, JF and RJ wrote the application for the approved ethical permit. RJ, PA, JF and CS created the study protocol. CS and RJ collected and prepared the data for statistical analysis. CS performed the statistical analysis. CS, RJ, JF and PA wrote the manuscript. All authors read and approved the final manuscript. CS and RJ contributed equally to this work.

\section{Acknowledgements}

Not applicable.

\section{References}

1. Hajibandeh S, Antoniou GA, Scurr JR, Torella F. Neuromuscular electrical stimulation for thromboprophylaxis: A systematic review. Phlebology. 2015;30(9):589-602.

2. Gniotek K, Frydrysiak M, Zięba J, Tokarska M, Stempień Z, editors. Innovative textile electrodes for muscles electrostimulation. 2011 IEEE International Symposium on Medical Measurements and Applications; 2011 30-31 May 2011.

3. Crema A, Malesevic N, Furfaro I, Raschella F, Pedrocchi A, Micera S. A Wearable Multi-Site System for NMES-Based Hand Function Restoration. IEEE Trans Neural Syst Rehabil Eng. 2018;26(2):428-40.

4. Williams KJ, Ravikumar R, Gaweesh AS, Moore HM, Lifsitz AD, Lane TR, et al. A Review of the Evidence to Support Neuromuscular Electrical Stimulation in the Prevention and Management of Venous Disease. Adv Exp Med Biol. 2017;906:377-86.

5. Lyons GM, Leane GE, Clarke-Moloney M, O'Brien JV, Grace PA. An investigation of the effect of electrode size and electrode location on comfort during stimulation of the gastrocnemius muscle. Med Eng Phys. 2004;26(10):873-8. 
6. Gobbo M, Gaffurini P, Bissolotti L, Esposito F, Orizio C. Transcutaneous neuromuscular electrical stimulation: influence of electrode positioning and stimulus amplitude settings on muscle response. Eur $\mathrm{J}$ Appl Physiol. 2011;111(10):2451-9.

7. Gobbo M, Maffiuletti NA, Orizio C, Minetto MA. Muscle motor point identification is essential for optimizing neuromuscular electrical stimulation use. J Neuroeng Rehabil. 112014. p. 17.

8. Botter A, Oprandi G, Lanfranco F, Allasia S, Maffiuletti NA, Minetto MA. Atlas of the muscle motor points for the lower limb: implications for electrical stimulation procedures and electrode positioning. Eur J Appl Physiol. 2011;111(10):2461-71.

9. Shaheen A, Javed N, Azam F, Liaquat A, Khan M, Alam SM, et al. Comparison of Bioelectrical Impedance and Navy Seal Formula to Measure Body Composition in Medical Students. Cureus. 2019;11(5):e4723.

10. Grimby G, Frändin K. On the use of a six-level scale for physical activity. Scand J Med Sci Sports. 2018;28(3):819-25.

11. Euler L, Juthberg R, Flodin J, Guo L, Ackermann PW, Persson NK. Textile Electrodes: Influence of Electrode Construction and Pressure on Stimulation Performance in Neuromuscular Electrical Stimulation (NMES). Annu Int Conf IEEE Eng Med Biol Soc. 2021;2021:1305-8.

12. Baker LL, McNeal DR, Benton LA, Bowman BR, Waters RL. Neuromuscular Electrical Stimulation. A Practical Guide. 3rd Edition. Downey, California: Los Amigos Research \& Education Institute; 1993.

13. Clarke Moloney M, Lyons GM, Breen P, Burke PE, Grace PA. Haemodynamic study examining the response of venous blood flow to electrical stimulation of the gastrocnemius muscle in patients with chronic venous disease. Eur J Vasc Endovasc Surg. 2006;31(3):300-5.

14. Hawker GA, Mian S, Kendzerska T, French M. Measures of adult pain: Visual Analog Scale for Pain (VAS Pain), Numeric Rating Scale for Pain (NRS Pain), McGill Pain Questionnaire (MPQ), Short-Form McGill Pain Questionnaire (SF-MPQ), Chronic Pain Grade Scale (CPGS), Short Form-36 Bodily Pain Scale (SF-36 BPS), and Measure of Intermittent and Constant Osteoarthritis Pain (ICOAP). Arthritis Care Res (Hoboken). 2011;63 Suppl 11:S240-52.

15. Altman DG. Practical Statistics for Medical Research. 1st ed. New York: Chapman and Hall/CRC; 1990. $624 \mathrm{p}$.

16. Praxitelous P, Edman G, Ackermann PW. Hemodynamics and tolerability of lower-limb intermittent pneumatic compression devices and neuromuscular stimulation. TRANSLATIONAL SPORTS MEDICINE. 2018;1(4):143-50.

17. Tucker A, Maass A, Bain D, Chen LH, Azzam M, Dawson H, et al. Augmentation of venous, arterial and microvascular blood supply in the leg by isometric neuromuscular stimulation via the peroneal nerve. Int 
J Angiol. 2010;19(1):e31-7.

18. Corley GJ, Birlea SI, Breen P, Olaighin G. Popliteal blood flow and plantar flexion force due to neuromuscular electrical stimulation (NMES) of the calf muscle pump are strongly associated with NMES intensity. Annu Int Conf IEEE Eng Med Biol Soc. 2009;2009:3051-4.

19. Glaviano NR, Saliba S. Can the Use of Neuromuscular Electrical Stimulation Be Improved to Optimize Quadriceps Strengthening? Sports Health. 2016;8(1):79-85.

20. Doucet BM, Lam A, Griffin L. Neuromuscular electrical stimulation for skeletal muscle function. Yale J Biol Med. 2012;85(2):201-15.

21. Christopoulos DG, Nicolaides AN, Szendro G, Irvine AT, Bull ML, Eastcott HH. Air-plethysmography and the effect of elastic compression on venous hemodynamics of the leg. J Vasc Surg. 1987;5(1):148-59.

22. Stefanou C. Electrical muscle stimulation in thomboprophylaxis: review and a derived hypothesis about thrombogenesis-the 4th factor. Springerplus. 2016;5(1):884.

23. Tsuda K, Takahira N, Ejiri M, Sakai K, Sakamoto M, Akamine A. Effect of resistance of the exercise band on the peak femoral vein velocity during active ankle flexion. Phlebology. 2020;35(3):176-83.

24. Toya K, Sasano K, Takasoh T, Nishimoto T, Fujimoto Y, Kusumoto Y, et al. Ankle positions and exercise intervals effect on the blood flow velocity in the common femoral vein during ankle pumping exercises. $J$ Phys Ther Sci. 2016;28(2):685-8.

25. Maffiuletti NA, Gondin J, Place N, Stevens-Lapsley J, Vivodtzev I, Minetto MA. Clinical Use of Neuromuscular Electrical Stimulation for Neuromuscular Rehabilitation: What Are We Overlooking? Arch Phys Med Rehabil. 2018;99(4):806-12.

26. Uhl JF, Gillot C. Anatomy of the veno-muscular pumps of the lower limb. Phlebology. 2015;30(3):18093.

27. Evans DR, Williams KJ, Strutton PH, Davies AH. The comparative hemodynamic efficacy of lower limb muscles using transcutaneous electrical stimulation. J Vasc Surg Venous Lymphat Disord. 2016;4(2):206-14.

28. Maffiuletti NA. Physiological and methodological considerations for the use of neuromuscular electrical stimulation. Eur J Appl Physiol. 2010;110(2):223-34.

29. Lachiewicz PF, Kelley SS, Haden LR. Two mechanical devices for prophylaxis of thromboembolism after total knee arthroplasty. A prospective, randomised study. J Bone Joint Surg Br. 2004;86(8):1137-41.

\section{Figures}




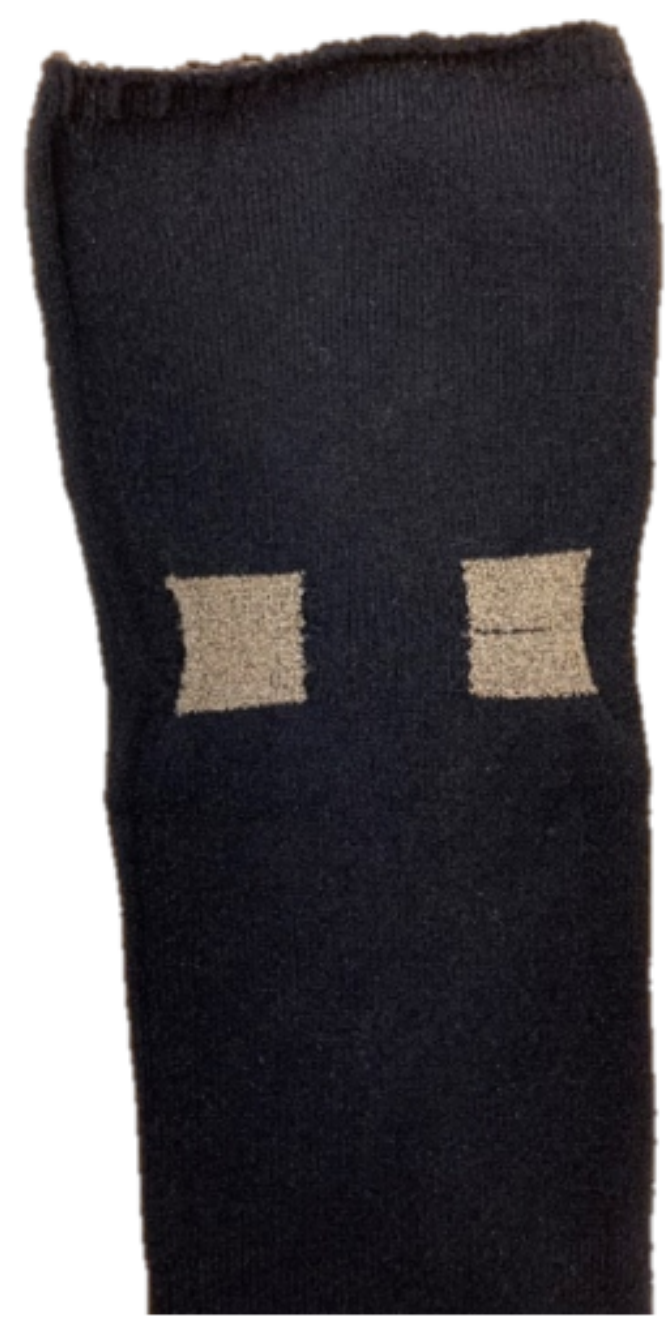

Figure 1

Textile electrodes. Sock with integrated textile electrodes used for the transverse textile electrode setup. 


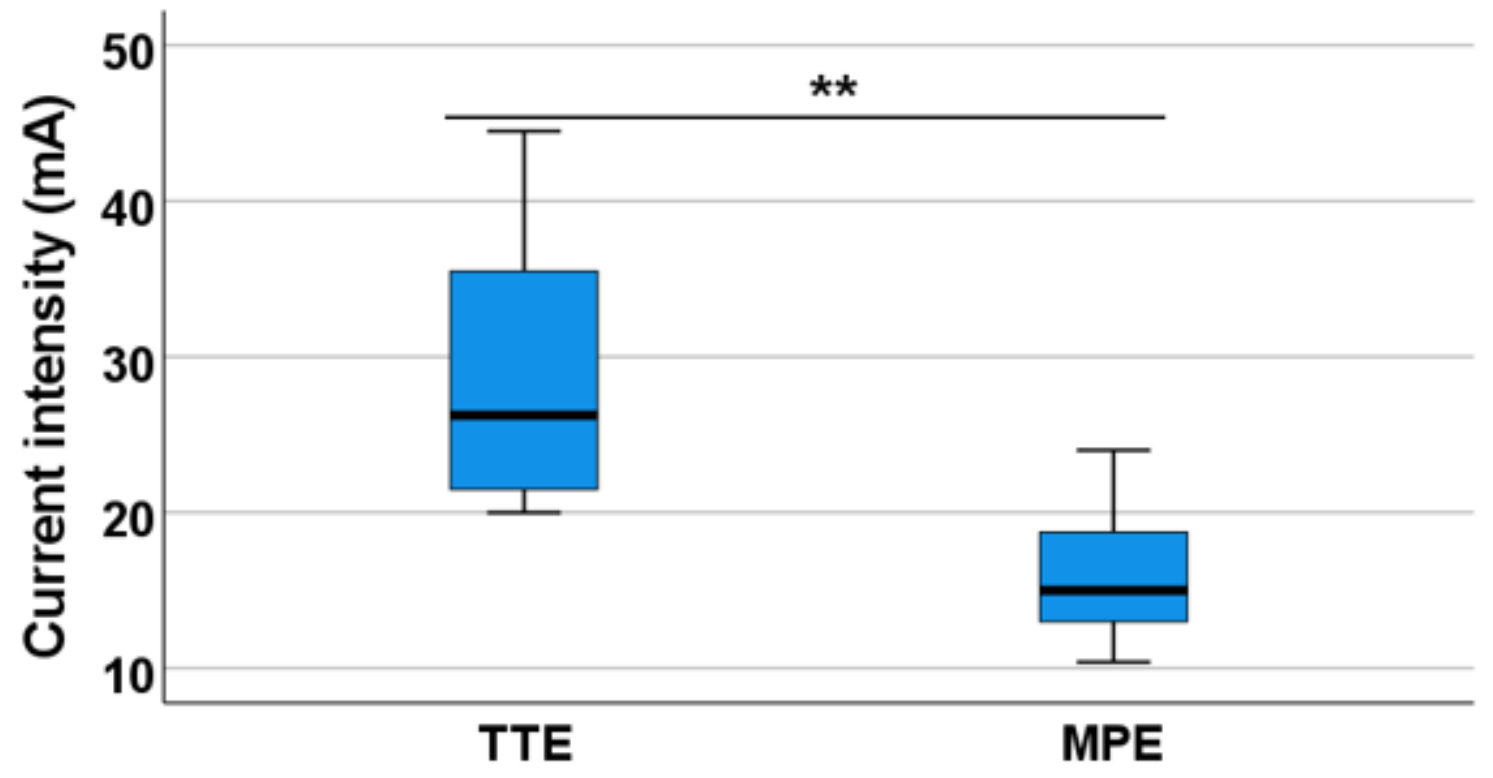

\section{Electrode setup}

Figure 2

Current intensity. Current intensity (mA) needed to reach threshold / displayed for both electrode setups. ** indicates $p<0.001$

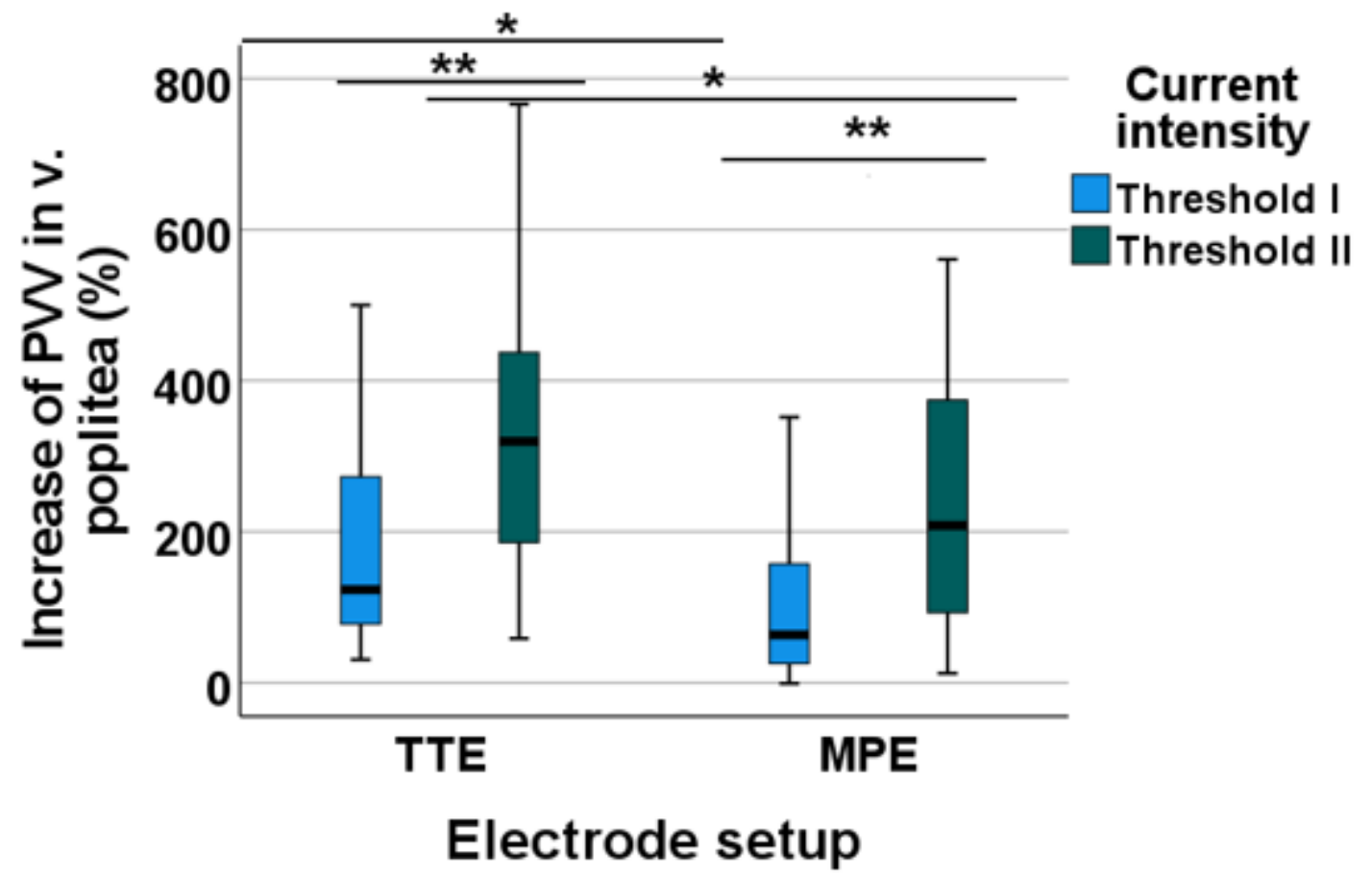

Figure 3

Hemodynamics in v. poplitea. Percentual increase of PVV from baseline to NMES threshold I and II with two electrode setups (TTE and MPE). The overlaying line connecting two boxplots indicates a difference, 
where * indicates a difference with $p<0.05$ and ** indicates a difference with $p<0.001 . P V V=$ Peak venous velocity. TTE $=$ transverse textile electrode setup. $\mathrm{MPE}=$ motor point electrode setup.

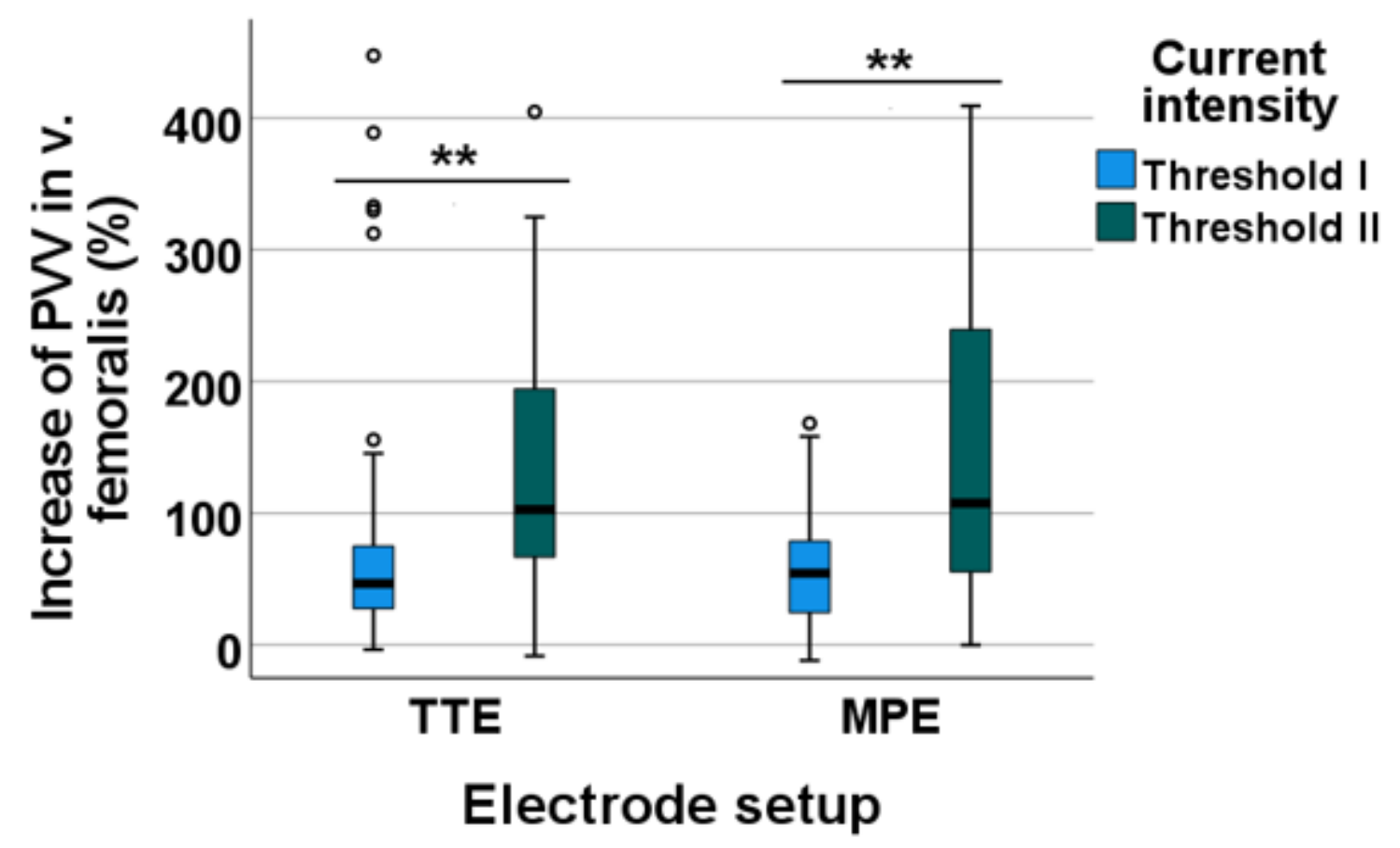

Figure 4

Hemodynamics in v. femoralis. Percentual increase of PVV from baseline to NMES threshold I and II with two electrode setups (TTE and MPE). The overlaying line connecting two boxplots indicates a difference, where * indicates a difference with $p<0.05$ and $* *$ indicates a difference with $p<0.001$. Circles represents outliers. $\mathrm{PVV}=$ Peak venous velocity. $\mathrm{TTE}=$ transverse textile electrode setup. MPE $=$ motor point electrode setup. 


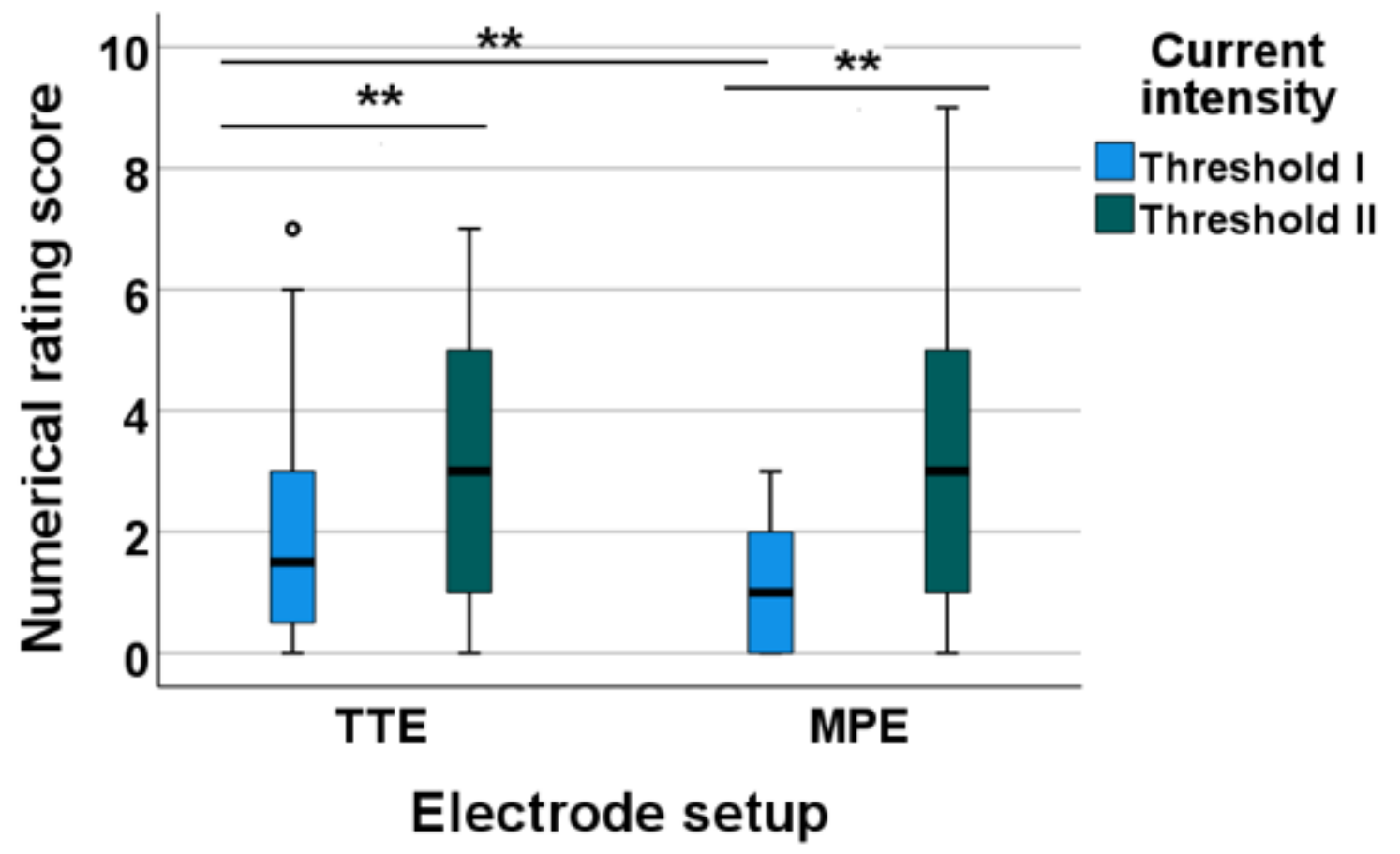

Figure 5

Numerical rating scale for the two electrode setups. Numerical rating scale (NRS) score for both electrode setups at both thresholds. $* *$ indicates $p<0.001$. Circles represents outliers. TTE $=$ transverse textile electrode setup. MPE = motor point electrode setup. 